\title{
Mitochondrial ROS produced in human colon carcinoma HCT116 cells reduces cell survival via autophagy
}

\author{
Eunji Gwak, Dasol Kim, Hui-Yun Hwang, and Ho Jeong Kwon* \\ ${ }^{1}$ Chemical Genomics Laboratory, Department of Biotechnology, College of Life Science and \\ Biotechnology, Yonsei University, Seoul 03722, Republic of Korea \\ *Correspondence: kwonhj@yonsei.ac.kr (H.J.K); Tel.: +82-2-2123-5883 (H.J.K.)
}

\begin{abstract}
Human colon carcinomas, including HCT116 cells, often exhibit high basal autophagic flux under nutrient deprivation or hypoxic conditions. Mitochondrial ROS (mROS) is known as a "molecular switch" for regulating the autophagic pathway, which is critical for directing cancer cell survival or death. In early tumorigenesis, autophagy plays important roles in maintaining cellular homeostasis and contributes to tumor growth. However, the relationships between mROS and the autophagic capacities of HCT116 cells are poorly understood. Ubiquinol cytochrome c reductase binding protein (UQCRB) has been reported as a biomarker of colorectal cancer, but its role in tumor growth has not been clarified. Here, we showed that UQCRB was overexpressed in HCT116 cells compared to CCD18co cells, a normal colon fibroblast cell line. Pharmacological inhibition of UQCRB reduced mROS levels, autophagic flux, and the growth of HCT116 tumors in a xenograft mouse model. We further investigated mutant UQCRB-overexpressing cell lines to identify functional links in UQCRB-mROS-autophagy. Notably, an increasing level of mROS caused by UQCRB overexpression released $\mathrm{Ca}^{2+}$ by activation of lysosomal TRPML1 channels. This activation induced transcription factor EB nuclear translocation and lysosome biogenesis, leading to autophagy flux. Collectively, our study showed that increasing levels of mROS caused by overexpression of UQCRB in human colon carcinoma HCT116 cells could be linked to autophagy for cell survival.
\end{abstract}

Keywords: UQCRB, mROS, Autophagy, Colorectal cancer, Lysosome

\section{Introduction}

Mitochondria contribute to malignant transformation of normal cells into neoplastic precursors by inducing the production of reactive oxygen species (ROS) [1]. Complex I and complex III of the electron transport chain have been recognized as the sites of superoxide production [2]. The mitochondrial ROS (mROS) can cause oncogenic DNA defects to accumulate and activate oncogenic signaling pathways [1]. Initially, mROS studies focused on the damaging effects of mROS, but recently, research has been shifting to determine whether mROS can act as a signaling molecule to support pro-growth responses in cells [3]. Targeting mitochondria-to-cell redox communication can alter activation of the cancer-promoting signaling pathway, thereby interfering in mitochondria-released oxidant signaling events, which could be a promising cancer therapy [4]. 
Ubiquinol-cytochrome c reductase-binding protein (UQCRB) is a $13.4-\mathrm{kDa}$ subunit of complex III in the mitochondrial respiratory chain [5] shown to play an important role in transporting electrons and maintaining the structure of complex III [6]. This subunit is a target protein of terpestacin, a small molecule that inhibits angiogenic responses [5]. Furthermore, the role of UQCRB as a component of the mitochondrial oxygen sensor has been suggested by modulating ROS production at complex III in response to hypoxia.

Despite advances in diagnosis and treatment, colorectal cancer (CRC) remains one of the major causes of cancer-related death in both men and women worldwide. In 2020, there were over 1 million new cases of CRC and over $\sim 500,000$ related deaths worldwide [7]. The relationship between UQCRB and CRC has been extensively studied. The UQCRB gene and protein levels are found at higher levels in CRC tissues than in adjacent non-tumor tissues [8]. In addition, UQCRB is more frequently highly expressed in CRC patients whose diseases are more advanced and who have poor pathological differentiation. The expression level of UQCRB-related circulating miR4435, which regulates the invasion of HCT116 cells and migration functions, is upregulated in HCT116 [8,9]. These results demonstrate that UQCRB expression is correlated with CRC progression, making it a biomarker for diagnosing CRC $[8,9]$.

Cancer cells uniquely reprogram their cellular activities to support rapid proliferation and migration, and to counteract metabolic and genotoxic stresses during cancer progression. In a previous study, we reported the rapid proliferation and invasion ability of mutant UQCRBexpressing cells (MT) [10]. In the present study, we found that UQCRB expression was significantly higher in HCT116 cells than in normal CCD 18co cells. Moreover, MT cells expressed a higher amount of autophagy marker protein than normal HEK293 cells, which contributed to the survival of the MT cells. Furthermore, a UQCRB inhibitor, A1938, suppressed the increased expression of autophagy marker protein in cells. Targeting mitochondrial UQCRB using the UQCRB inhibitor could therefore be a possible treatment for CRC patients.

\section{Results}

\subsection{The UQCRB-overexpressing CRC cell line HCT116 is sensitized by A1938-induced cell death.}

UQCRB was discovered as a target protein of the anti-angiogenic natural small molecule terpestacin [5]. Overexpression of UQCRB causes the production of mROS, and inhibition of UQCRB decreases mROS generation in various cancer cell lines, including HT1080, HepG2, and U87MG [5,11-13]. Although the relationship between UQCRB and CRC has been studied [8], the underlying mechanism of inhibition of UQCRB-induced cell death remains unknown. To study the function of UQCRB in CRC, we investigated UQCRB expression levels in HCT116 cells by western blot analysis using anti-UQCRB antibodies and found that endogenous UQCRB levels in CRC cells were higher than those in normal CCD18Co cells (Fig. 1A). Because the basal level of ROS has been shown to be increased in cancer cells when compared with normal cells [14], we investigated whether inhibition of UQCRB regulates ROS generation in HCT116 cells. Notably, the high mROS levels in HCT116 cells were suppressed by treatment with A1938, a UQCRB inhibitor (Figs. 1B and 1C). 
Prior to characterizing the effect of the underlying mechanism of UQCRB inhibition activity, we determined the effects of A1938 on cell proliferation and viability of HCT116 cells. The HCT116 cells were treated with various doses of A1938 for 0-72 h, and their cell growth was measured using a 3-(4,5-dimethylthiazol-2-yl)-2,5-diphenyltetrazolium bromide (MTT) colorimetric assay. The results showed that various doses of A1938 affected the growth of HCT116 cells, with an effective concentration starting at $30 \mu \mathrm{M}$ (Fig. 1D). In addition, trypan blue staining showed that HCT 116 cells treated with A1938 in medium containing 10\% serum did not show cytotoxicity up to $50 \mu \mathrm{M}$ (Fig. 1E). A concentration of $30 \mu \mathrm{M}$ A1938 was therefore used in subsequent studies.

Several types of CRC-derived cells, including HCT116 cells, often exhibit high basal autophagic flux under nutrient deprivation or hypoxia [15-18]. For these reasons, we investigated whether the UQCRB inhibitor A1938 could affect the growth of cancer cells by inhibiting autophagy. At doses above $20 \mu \mathrm{M}$ under serum-starved conditions, A1938 had a cytotoxic effect on HCT116 cells of over $67 \%$. To determine if the decreased cell growth and viability were the results of autophagy inhibition, HCT116 cells were treated with the autophagy inhibitor, chloroquine (CQ) (Fig. 1D). Co-treatment with A1938 and CQ further reduced cell viability, indicating that autophagy played a role in the survival of HCT116 cells. Taken together, these results demonstrated that autophagy was suppressed by inhibiting UQCRB activity using a UQCRB inhibitor, which led to increased cytotoxicity. Cell viability significantly decreased under serum-starvation conditions as a result of increased autophagy activation involving recycling of cell components to facilitate survival in a nutrient deprived environment. These results suggested that targeting UQCRB activity by A1938, a UQCRB inhibitor, could inhibit autophagy in CRC cells.

A)

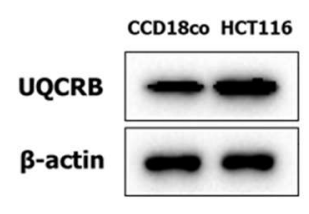

D)

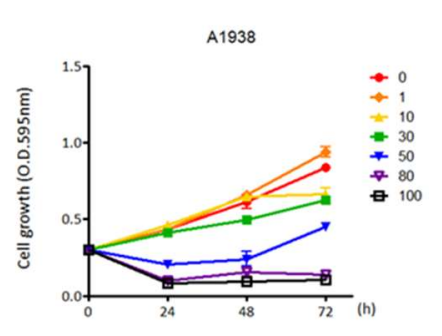

B)

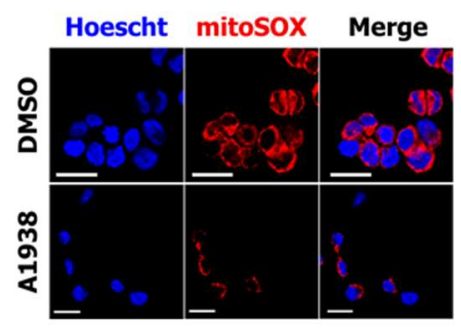

E)

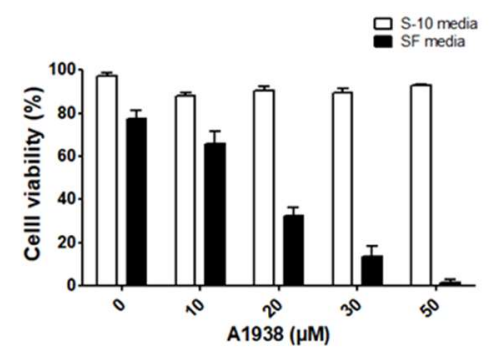

C)

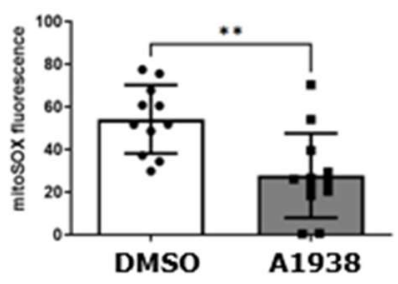

F)

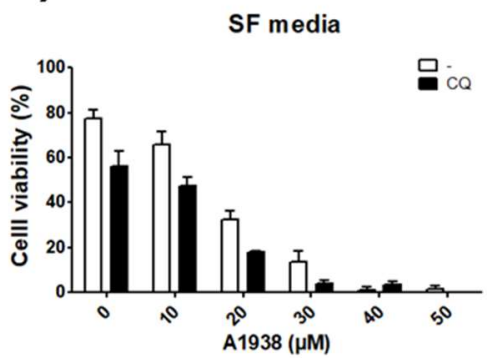

Figure 1. A1938-induced cell death is sensitized in HCT116 cells. (A) Expression levels of UQCRB in CCD18co normal human colon cells and HCT116 CRC cells. $\beta$-actin was used as an internal control. (B) Confocal microscopy images of MitoSOX Red fluorescence showing mROS generated in HCT116 cells. (C) The mean \pm SD of cells (n $=11$ ) treated with $30 \mu \mathrm{M}$ A1938 for $4 \mathrm{~h}$. Scale bar, $20 \mu \mathrm{m}$. (D) HCT116 cell viability after treatment with $0,1,10,30,50,80$, or $100 \mu \mathrm{M}$ A1938 as assessed using the MTT assay. 
(E) The viability of A1938-treated HCT116 cells using concentrations of 0, 10, 20, 30, or $50 \mu \mathrm{M}$ in the trypan blue assay. (F) HCT116 cell viability as measured using ImageJ software after treatment with $0-80 \mu \mathrm{M}$ A1938 in the presence or absence of $5 \mu \mathrm{M}$ chloroquine for 3 days in serum-free medium. Statistical significance was assessed using the Student's $t$-test. ${ }^{* *} \mathrm{P}<0.01$.

\subsection{Autophagy is induced in mutant UQCRB-overexpressing cells (MT).}

The overexpression of mutant UQCRB causes the rapid production of mROS and abnormal mitochondrial morphology, which is characterized by significant swelling [10]. We therefore determined the effect of mROS production caused by UQCRB overexpression on autophagy during cell proliferation. First, we examined whether the MT stable cell line retained its characteristics. Immunoblotting using anti-UQCRB and anti-Myc antibodies showed that validated MT cells overexpressed UQCRB, which is consistent with the results of a previous study (Fig. 2A). Furthermore, mROS levels were higher in MT cells than in control HEK293 cells (Supplementary Fig. 1), which suggested that the production of large amounts of mROS in MT cells induced autophagy in a similar way as treatment with exogenous oxidants.

Next, we confirmed the presence of autophagy markers LC3 and p62 in MT cells using immunoblotting. During autophagy, LC3-I converted to LC3-II, which is a key component of autophagosomes. In ubiquitin-dependent autophagy, autophagic cargo receptor proteins, such as p62 act as degradation substrates of the autophagic membrane, so that p62 protein levels can be increased while autophagy occurs [19]. MT cells had higher LC3-II and p62 levels than normal HEK293 cells, both when treated with serum and when starved (Figs. 2B-2D). Next, autophagy induction was examined by immunocytochemistry using LC3 antibodies. HEK293 and MT cells were incubated in SF medium for $24 \mathrm{~h}$, after which LC3 puncta were only observed in MT cells (Fig. 2E). This result demonstrated that UQCRB overexpression contributed to mROS production, making cells sensitive to serum-free, nutrient-deprivation environments that that lead to autophagy.

Autophagy is important to the constitutive turnover of intracellular components and for the elimination of potentially damaging or abnormal intracellular components [20]. To investigate autophagic flux, CQ, an inhibitor of autophagy that impairs autophagosome fusion with lysosomes, was used to treat HEK293 and MT cells [21]. First, HEK293 and MT cells were treated with $5 \mu \mathrm{M}$ of CQ for $0-6 \mathrm{~h}$ to investigate their autophagy and autophagic flux kinetics. In MT cells, LC3-II and p62 increased in a time-dependent manner, whereas p62 protein levels did not change or even decreased over $4 \mathrm{~h}$ in CQ-treated HEK293 cells (Fig. 2F). After treating with CQ for $6 \mathrm{~h}$, p62 levels in MT cells were significantly higher than those in HEK293 cells. CQ treatment blocked autophagosome degradation, resulting in higher levels of LC3-II in both HEK293 and MT cells. However, the densitometric values of LC3-II and LC3-I, indicated that MT cells exhibited a greater LC3-II conversion rate than control cells (Fig. 2G). To further validate the effect of UQCRB on autophagy flux, a double-tagged mRFP-GFP-LC3 plasmid was used to visualize the transition from neutral autophagosomes to acidic autolysosomes based on the different $\mathrm{pH}$ stabilities of mRFP-LC3 and GFP-LC3. GFP fluorescence is unstable in acidic compartments, whereas mRFP fluorescence is relatively stable, even in the acidic environment of lysosomes [22]. Accordingly, autophagic flux was monitored according to the decrease in co-localization of green and red fluorescence, which appeared yellow to indicate the presence of autophagosomes and an increase 
in red fluorescence, which reflects the presence of autolysosomes. We saw little yellow or red puncta fluorescence in control cells, but red puncta fluorescence was observed in MT cells (Fig. 2H). HEK293 cells also had diffused LC3 with puncta that were difficult to discern (Fig. 2E). Together, these results indicated that the normal expression of UQCRB could not actively induce autophagy, suggesting that the overexpression of UQCRB induced more autophagy flux.
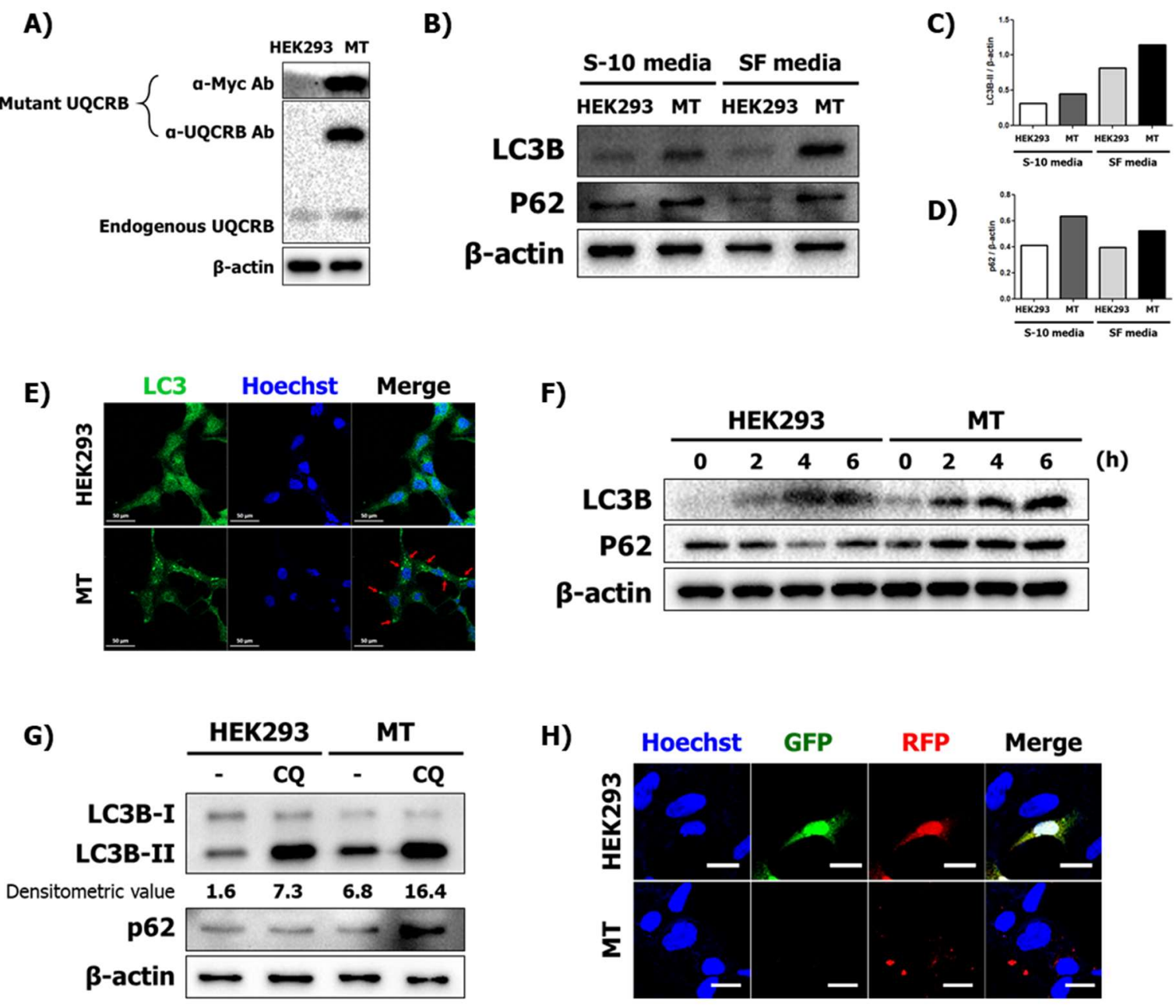

Figure 2. Autophagy is induced in MT cells. (A) Mutant UQCRB-expressing cell lines were examined by western blotting using anti-UQCRB and anti-Myc antibodies. (B) Representative images and (C) intensities of LC3B and (D) p62 immunoblotting bands normalized to $\beta$-actin. HEK293 and MT cells treated with either $10 \%$ serum-containing medium or serum-free medium for $24 \mathrm{~h}$ and then analyzed by western blot analysis using antibodies against LC3B and p62. (E) Confocal microscopy images of HEK293 and MT cells treated with serum-free medium for $24 \mathrm{~h}$ after immunostaining with anti-LC3 antibody. Scale bar, $50 \mu \mathrm{m}$. Data are presented as the mean $\pm \mathrm{SD}$; statistical significance was assessed using an unpaired $t$-test. (F) Western blot analysis results of LC3B and p62 levels in cells after treatment with $5 \mu \mathrm{M}$ chloroquine (CQ) treatment for $0-6 \mathrm{~h}$. (G) 
HEK293 and MT cells were treated with either D.W. or CQ for $6 \mathrm{~h}$. Cell extracts were subjected to western blotting using LC3B and p62 antibodies. The densitometric value of LC3-II was quantitated based on western blot data and normalized to LC3-I levels. (H) Confocal microscopy images of HEK293 and MT cells transfected with mRFP-GFP-LC3 for $24 \mathrm{~h}$. Scale bar, $20 \mu \mathrm{m}$.

\subsection{The effects of UQCRB overexpression on activation of TRPML1 $\mathrm{Ca}^{2+}$ channels and transcription factor EB (TFEB) via mROS.}

The mROS is known to directly and specifically activate the lysosomal $\mathrm{Ca}^{2+}$ channel, TRPML1, inducing lysosomal $\mathrm{Ca}^{2+}$ release that activates calcineurin [23]. We hypothesized that increasing mROS levels by overexpressing UQCRB could trigger lysosomal TRPML1 channels and induce lysosomal $\mathrm{Ca}^{2+}$ release. HEK293 and MT cells were therefore transfected with GCaMP3-ML1 encoding a lysosome-targeted $\mathrm{Ca}^{2+}$ probe. The structure of the GFP tag on the channel protein in the outer lysosomal membrane shifts when this $\mathrm{Ca}^{2+}$ probe captures $\mathrm{Ca}^{2+}$ ions [24]. Without any other treatment, MT cells released higher $\mathrm{Ca}^{2+}$ levels than HEK293 cells. In addition, the TRPML1 channel agonist, ML-SA1, induced more $\mathrm{Ca}^{2+}$ release in MT cells than in HEK293 cells. Accordingly, increased mROS levels in MT cells resulted in the activation of lysosomal TRPML1 more frequently by ML-SA1 (Fig. 3A and 3B), showing that overexpression of UQCRB indirectly regulated the lysosomal $\mathrm{Ca}^{2+}$ ion channel, causing $\mathrm{Ca}^{2+}$ to be released from lysosomes into the cytosol.

TFEB-nuclear translocation can be stimulated by release of lysosomal $\mathrm{Ca}^{2+}$, and the $\mathrm{Ca}^{2+}$ dependent phosphatase, calcineurin [24]. TFEB is normally maintained in an inactive state by phosphorylation in the cytosol. However, TFEB translocation into the nucleus can activate autophagy- and lysosome-related genes as a transcription factor [25]. Thus, TFEB localization could be physiologically related to TFEB activation. In addition, ROS levels are elevated during starvation [26], which activates TFEB and promotes autophagy [24].

To investigate whether TFEB translocation was related to UQCRB in affecting mROS levels, the enhanced green fluorescence protein (EGFP)-TFEB plasmid was transfected into HEK293 and MT cells to directly observe the nuclear translocation of TFEB. When treated with serum, TFEB translocated into the nucleus in three out of 20 HEK293 cells (15\%) but into 19 of 37 MT cells (51\%) (Fig. 3C and 3D). However, A1938 treatment reduced the mROS production levels in MT cells (Supplementary Fig. 1). After A1938 treatment, the nucleus-cytoplasm ratio of TFEB was 35\% lower than in MT cells treated with dimethyl sulfoxide (DMSO) (Fig. 3C and 3D). Collectively, these results demonstrated that UQCRB inhibition reduced mROS production through the regulation of lysosomal $\mathrm{Ca}^{2+}$-mediated TFEB signaling. 
A)

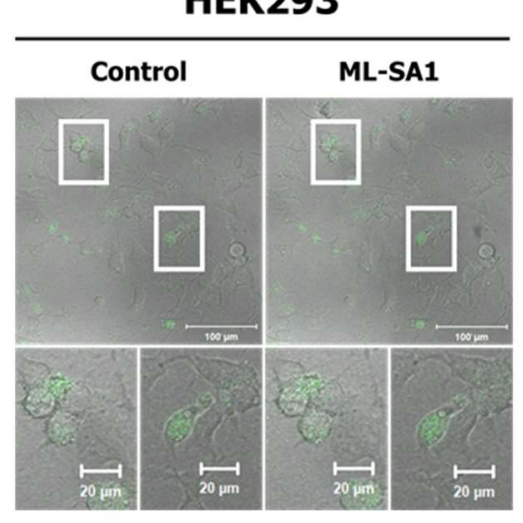

C)

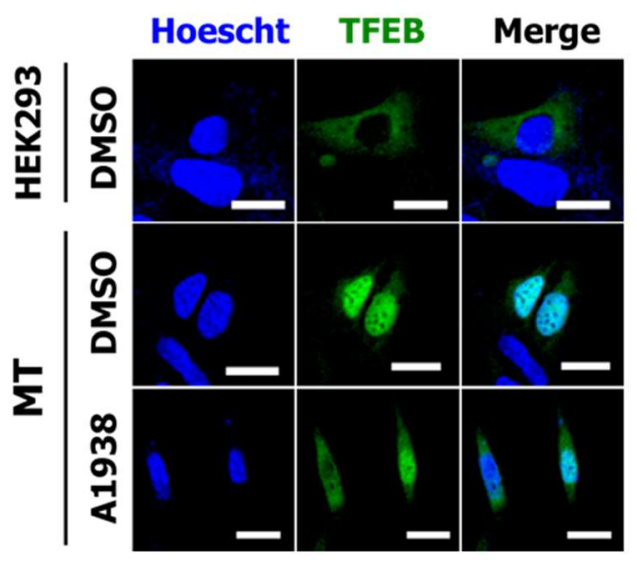

B)

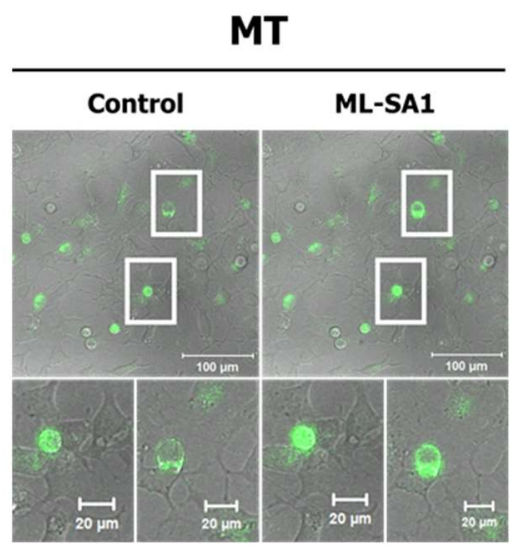

D)

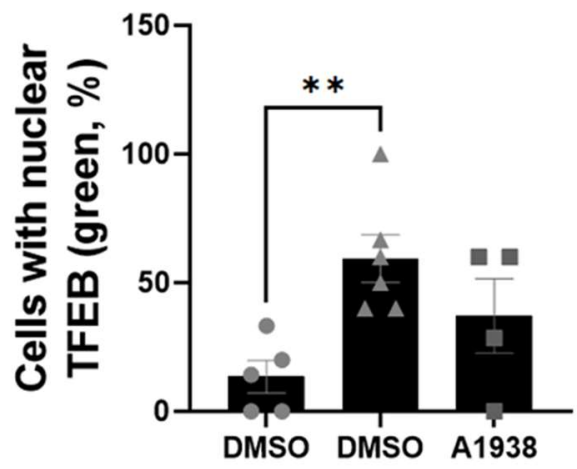

Figure 3. TRPML1-induced lysosomal $\mathrm{Ca}^{2+}$ release and TFEB activation are increased in MT cells. HEK293 and MT cells were transfected with GCaMP3-TRPML1 encoding a lysosome-specific $\mathrm{Ca}^{2+}$ probe, $40 \mu \mathrm{M}$ ML-SA1, for $120 \mathrm{~s}$. (A) Confocal microscopy images of GCaMP green fluorescence showing HEK293 and (B) MT cells. ML-SA1 treatment for $120 \mathrm{~s}$. (C) Confocal microscopy images of HEK293 and MT cells transfected with EGFP-TFEB and treated with the DMSO control or A1938 for $6 \mathrm{~h}$. (D) The number of cells with a nuclear (Nuc) or cytoplasmic (Cyt) TFEB localization ratio is represented in the graph. Scale bar, $20 \mu \mathrm{m}$. Statistical significance was assessed using Student's $t$-test. ${ }^{* *} \mathrm{P}<0.01$.

\subsection{The effect of A1938 on increased lysosome activity in MT cells}

The role of lysosomes in degradation and recycling involves a cellular housekeeping function [24]. Intracellular substrates are delivered to the lysosome by the autophagic pathway by fusing autophagosomes with lysosomes. TFEB is a master regulator of lysosomal and autophagic functions and of energy metabolism [24]. Thus, we examined lysosome biogenesis and activity using LysoTracker Red (LTR) and acridine orange (AO), to stain acidic compartments, such as 
lysosomes.

After LTR staining, red puncta showed the locations of lysosomes [27]. DMSO-treated MT cells had higher levels of acidic vesicles than DMSO-treated normal HEK293 cells. Specifically, an average of nine red puncta were detected in HEK293 cells, while an average of 39 were detected in MT cells. A1938 treatment reduced the number of red puncta to an average of 17 in MT cells (Fig. 4A and 4B).

Lysosomes play a role in cell component degradation by maintaining an acidic environment with a $\mathrm{pH}$ of approximately 5 to facilitate functioning of hydrolytic enzymes. Treating MT cells with A1938 caused their AO fluorescence intensity to significantly decrease, which indicates that lysosome function was inhibited (Fig. 4C).

Because TFEB is a transcriptional regulator of lysosome biogenesis [28], we investigated how UQCRB was related to mROS during lysosome biogenesis. LAMP1 is used as a lysosomal marker, and LAMP1-positive organelles are considered as lysosomal compartments [29]. The expression of the lysosomal protein LAMP1 was higher in MT cells than in HEK293 cells, and A1938 treatment decreased lysosomal biogenesis (Fig. 4D and 4E). These results suggest that an increased level of mROS produced by UQCRB overexpression induced lysosomal biogenesis through TRPML1-TFEB activation, and that this process was regulated by an UQCRB inhibitor.

A)

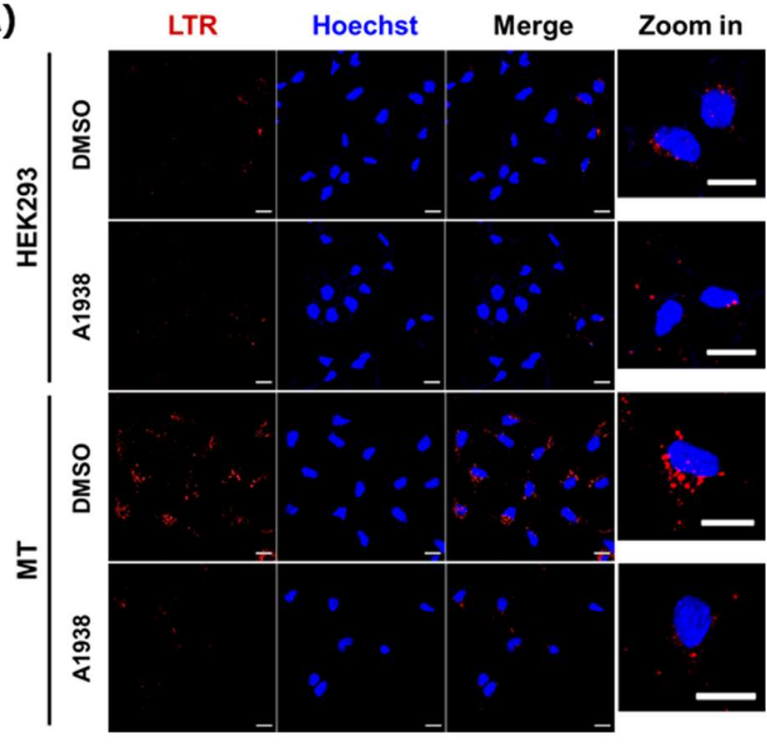

B)

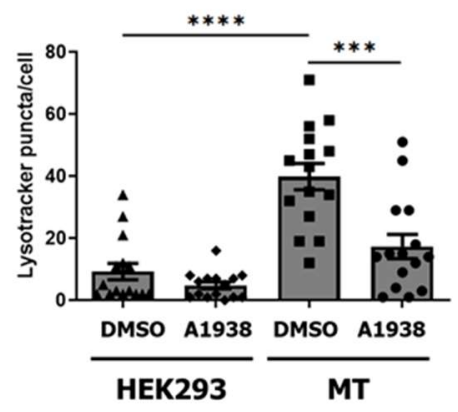

C)
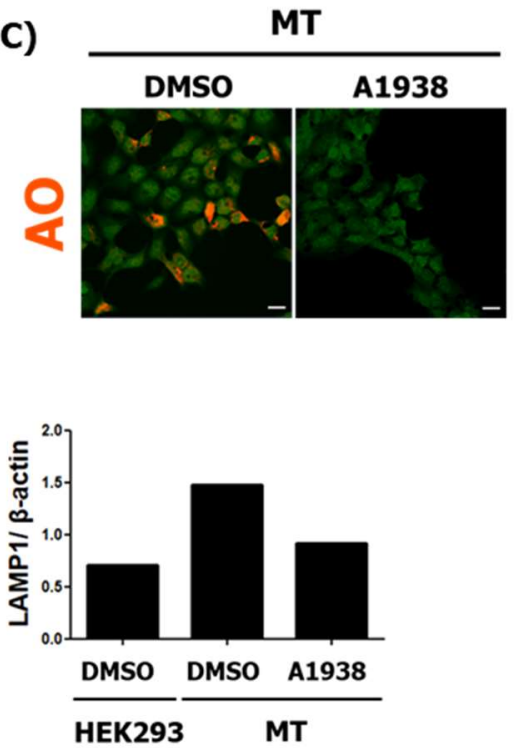
Figure 4. Lysosome activity in MT cells with or without A1938 treatment. (A) Confocal microscopy images and (B) Numbers of acidic lysosome red puncta were counted in HEK293 and MT cells $(n=15)$. The cells were treated with DMSO as a control or $30 \mu \mathrm{M}$ A1938 for $24 \mathrm{~h}$ and then stained with LTR. Scale bar, $20 \mu \mathrm{m}$. Statistical significance was assessed using Student's $t$-test. ${ }^{* * *} \mathrm{P}<0.0001,{ }^{* * *} \mathrm{P}<0.001$. (C) Confocal microscopy images of MT cells treated with DMSO as a control or $30 \mu \mathrm{M}$ A1938 for $24 \mathrm{~h}$, then the live cells were stained with $2 \mu \mathrm{g} / \mathrm{mL}$ acridine orange for $25 \mathrm{~min}$ and fixed. Scale bar, 20 $\mu \mathrm{m}$. (D) Images of HEK293 and MT cell extracts treated with the DMSO control or 30 $\mu \mathrm{M}$ A1938 for $24 \mathrm{~h}$ in serum-free medium, then subjected to western blot analysis using antibodies against LAMP1. (E) Intensities of the LAMP1 immunoblot bands were normalized to $\beta$-actin expression.

\subsection{The effect of A1938 on autophagy flux by suppression of mROS generation}

ROS can function as a signaling molecule that induces autophagy, which tends to degrade dysfunctional mitochondria. Zhang et al. evaluated the effect of mROS on autophagy induction using the exogenous oxidants, carbonyl cyanide $m$-chlorophenylhydrazone and rotenone [23]. We therefore evaluated the effect of A1938 on autophagy by suppressing mROS production in MT cells. MT cells treated with A1938 exhibited increased LC3B-II and p62 protein levels when compared with MT cells treated with DMSO (Fig. 5A-5C). To evaluate the role of UQCRB in this activity, the lysosomal inhibitor, CQ, was used. The amounts of LC3-II and p62 accumulating in CQ-treated cells were higher than in DMSO-treated control cells. However, in A1938 and CQ cotreated cells, LC3-II levels were unchanged and p62 levels decreased (Fig. 5D). In cells expressing mRFP-GFP-LC3, A1938 treatment resulted in decreased red fluorescence in MT cells (Fig. 5E and $5 \mathrm{~F}$ ), indicating that inhibiting the production of mROS suppressed autophagy induction (Supplementary Fig. 1). Overall, autophagosomes likely accumulated due to inhibition of autophagic degradation, which indicates that A1938 decreased autophagy flux. 
A)

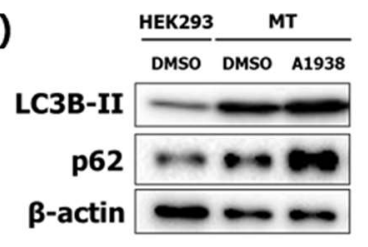

B)

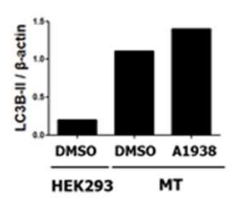

C)

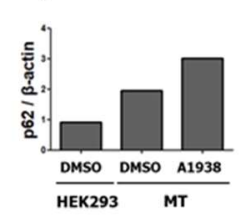

E)

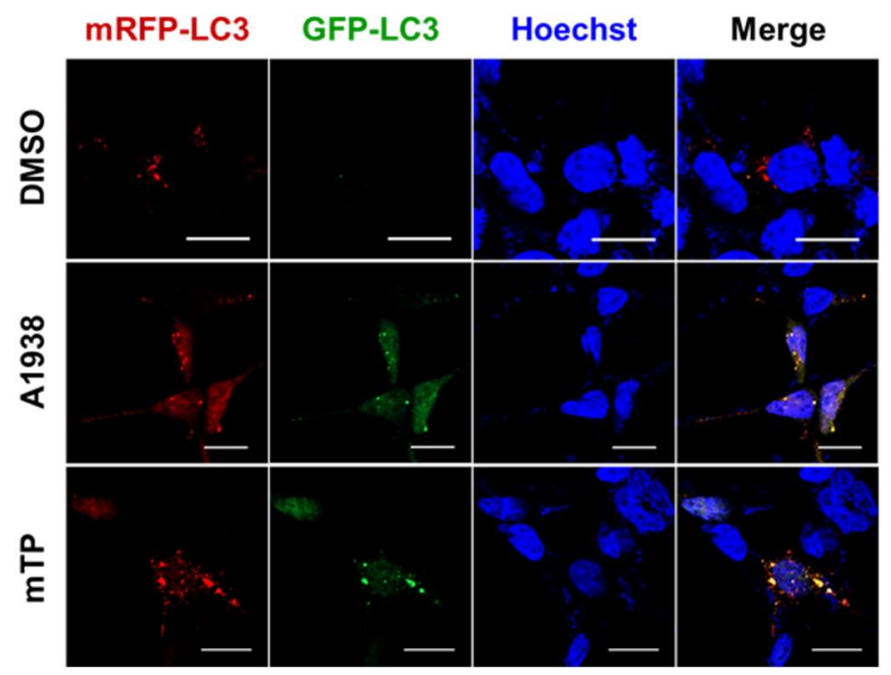

D)

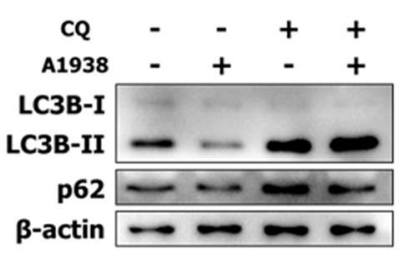

F)

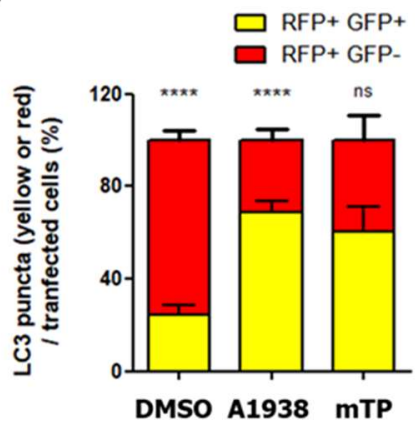

Figure 5. A1938 reduces LC3B expression and regulates autophagy flux by suppression of mROS generation in MT cells. (A) Representative images and (B-C) intensities of LC3B and p62 immunoblot bands normalized to $\beta$-actin in cells treated with A1938 for $24 \mathrm{~h}$ in serum-free medium. (D) A1938 treatment with or without chloroquine (CQ) in MT cells. Cell extracts of MT cells subjected to western blot analysis using antibodies against LC3B and p62. A total of $30 \mu \mathrm{M}$ A1938 and $5 \mu \mathrm{M}$ CQ was used to treat the cells for $6 \mathrm{~h}$. (E) Confocal microscopy images of HEK293 and MT cells transfected with mRFP-GFP-LC3 and treated with DMSO, A1938, or mTP for $24 \mathrm{~h}$. Scale bar, $20 \mu \mathrm{m}$. Quantification of data is shown in $(\mathrm{F})$. Values are the means $\pm \mathrm{SEM} ; \mathrm{n}=10$ cells, $\mathrm{ns}, \mathrm{P}>$ $0.05,{ }^{* * * *} \mathrm{p}<0.001$.

\subsection{HCT116 is sensitized by A1938-induced autophagy regulation.}

Cancer cells are usually able to tolerate stressed environments through autophagy, which removes ROS and damaged proteins, and maintains mitochondrial functioning and cellular metabolism, to promote survival under stress [14]. As shown in Fig. 1, A1938-induced cell death was accompanied by serum-free, nutrient-deprived environmental conditions in HCT116 cells. To confirm that A1938-mediated autophagy inhibition affected HCT116 survival under serum starvation conditions, treatment with A1938 and mTP in serum-free conditions for $24 \mathrm{~h}$ resulted in increased yellow fluorescence in HCT116 cells, indicating that inhibiting the production of mROS suppressed autophagy flux (Fig. 6A and 6B). In addition, HCT116 cells were treated with A1938 for $24 \mathrm{~h}$ under starvation conditions, which dramatically increased their LC3B levels (Fig. 6C). These results indicate that A1938 inhibited autophagy flux in HCT116 by decreasing the 
expression of mROS.

In this study, the inhibition of UQCRB protein by A1938 reduced autophagy and lysosomal activity in MT cells (Figs. 4 and 5). We therefore determined whether TFEB nuclear translocation and lysosomal activity could be regulated by treating HCT116 cells with the UQCRB inhibitor. Consistently, TFEB translocation into the nucleus was abolished after treatment of HCT116 cells with A1938 and mTP, indicating that TFEB activation was regulated by mROS production (Fig. $6 \mathrm{D}$ and $6 \mathrm{E})$.

When HCT116 cells were treated with A1938 for $24 \mathrm{~h}$ under starvation conditions and stained with AO, there were significantly fewer acidic vesicles in A1938-treated HCT116 cells than in DMSO-treated HCT116 cells, indicating that A1938 prevented lysosome activity by inhibiting UQCRB activity (Fig. 6F and 6G). Furthermore, expression of the lysosomal protein LAMP1 was decreased after treatment with A1938 (Fig. 6H). Taken together, these results indicate that inhibiting UQCRB protein reduced mROS levels, which decreased lysosomal activity. 
A)

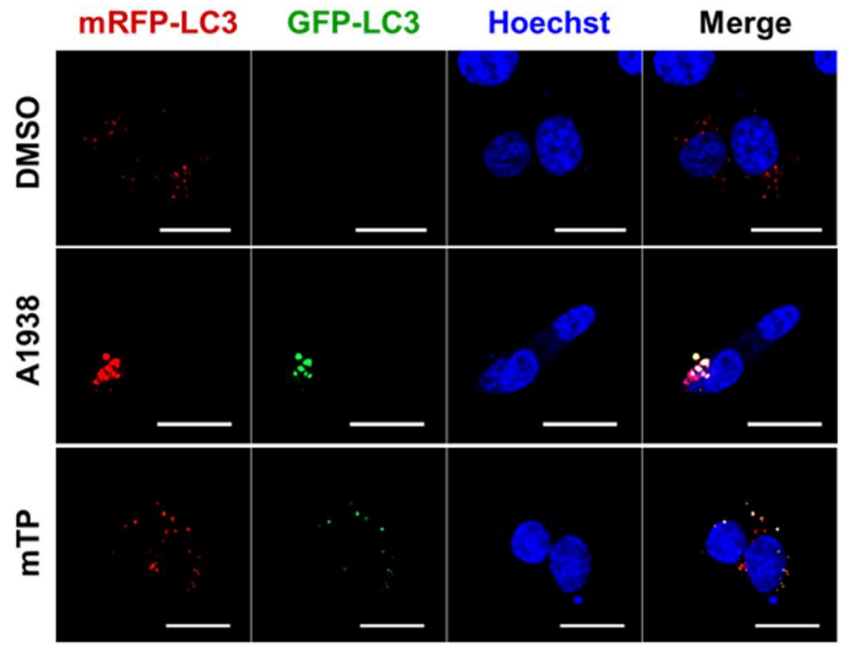

D)

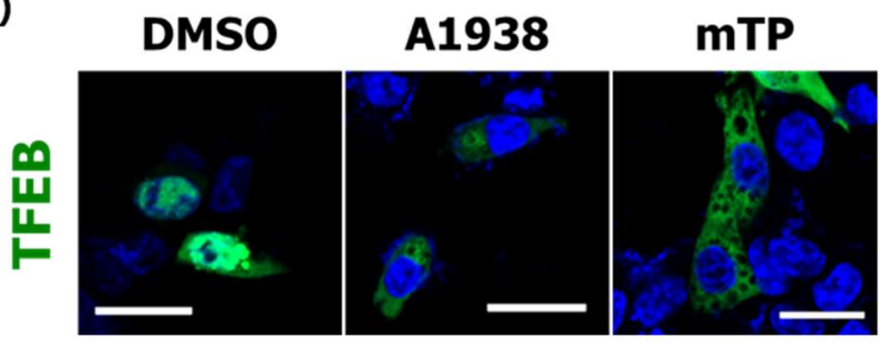

F)

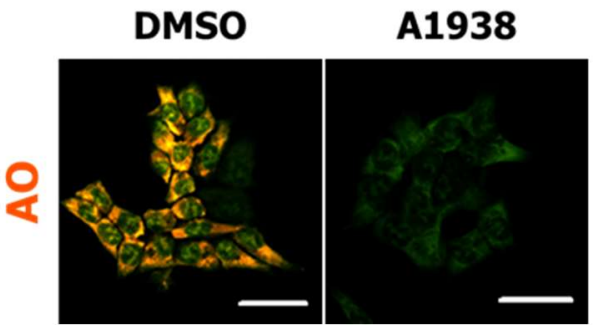

G)

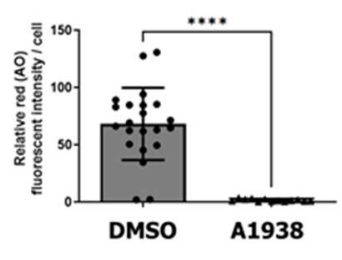

B)

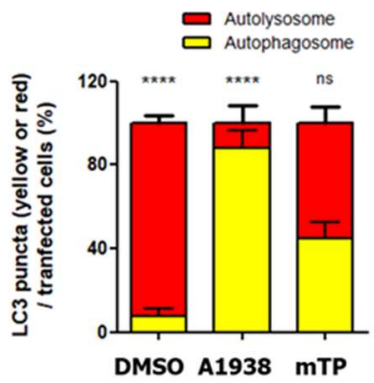

C)

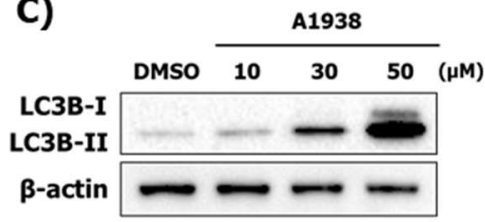

E)

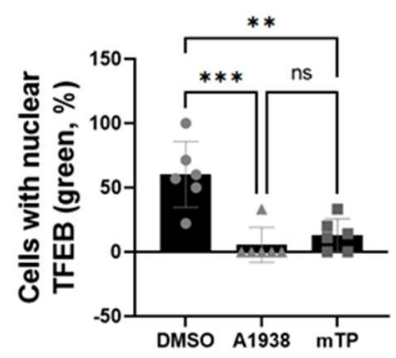

H)

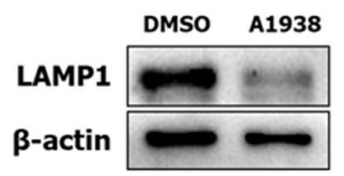

Figure 6. A1938 reduces autophagy through down-regulation of mROS in HCT116 cells (A) Confocal microscopy image of HCT116 cells transfected with mRFP-GFP-LC3 and treated with DMSO, A1938, or mTP for $24 \mathrm{~h}$. Scale bar, $20 \mu \mathrm{m}$. Quantification of data is shown in (B). (C) HCT 116 cells treated with various concentrations of A1938 for $24 \mathrm{~h}$ in serum-free medium. The resulting cell extracts were subjected to western blot analysis using antibodies against LC3B. (D) Confocal microscopy images of HCT116 cells transfected with GFP-TFEB and treated with the DMSO control or A1938 for $6 \mathrm{~h}$. (E) The number of cells with a nuclear (Nuc) or cytoplasmic (Cyt) TFEB localization ratio is represented in the graph. (F) HCT116 cells were treated with DMSO control or $30 \mu \mathrm{M}$ A1938 for $24 \mathrm{~h}$. Live cells were stained with $2 \mu \mathrm{g} / \mathrm{mL}$ acridine orange (AO) for $25 \mathrm{~min}$, fixed, and examined by confocal fluorescence microscopy. Confocal fluorescence 
microscopy images of HCT116 cells treated with A1938 in RPMI medium containing 10\% serum. (G) The average AO intensity per HCT116 cell treated with A1938 in RPMI medium containing $10 \%$ serum $(\mathrm{n}>10)$. (H) HCT116 cells treated with $30 \mu \mathrm{M}$ A1938 for $24 \mathrm{~h}$ and then subjected to western blot analysis using antibodies against LAMP1. Scale bar, $20 \mu \mathrm{m}$. Statistical significance was assessed using Student's $t$-test. ns, $\mathrm{P}>0.05$, ${ }^{* *} \mathrm{P}<0.01,{ }^{* * *} \mathrm{P}<0.001,{ }^{* * * *} \mathrm{P}<0.0001$.

\section{Discussion}

Mitochondria are important to oxygen-sensing capabilities of cells [30]. UQCRB is especially an important oxygen sensor in hypoxia-induced [5], and VEGF-induced angiogenesis [31] plays a key role in this process. We previously reported that UQCRB overexpression affected mitochondrial morphology [10] and that mitochondrial dynamics were strongly associated with the clearance of damaged mitochondria [32]. Recent reports have also indicated that sustained elevation of ROS levels contributed to stress signaling in cells, which may damage mitochondria and lead to autophagy $[4,32,33]$. Although the associations between ROS and autophagy have been studied, these studies mainly used exogenous oxidants to increase mROS levels, which did not reflect a natural environment [32-34].

Although the connection between ROS and autophagy has been well studied [23], the role of UQCRB in autophagy is largely unknown. In the present study, we determined the role of UQCRB protein overexpression in autophagy, with respect to its effect on mROS production. Previously, Chang et al. generated a novel protein transduction domain (PTD)-conjugated wild type UQCRB fusion protein, and treatment with PTD-UQCRB generated mROS without cytotoxicity [12]. To further analyze autophagy, mutant expressing UQCRB and HCT116 cells were used in this study. The results are consistent with those of previous studies, which showed that the overexpression of UQCRB induced mROS generation, but this generation was reduced by treatment with the UQCRB inhibitor A1938 (Supplementary Fig. 1). We also showed that overexpression of UQCRB promoted autophagy flux, spontaneously induced $\mathrm{Ca}^{2+}$ release by TRPML1 lysosomal $\mathrm{Ca}^{2+}$ ion channels, and induced TFEB translocation (Fig. 3). These results are consistent with those of previous studies showing that elevation in mROS levels led to TRPML1 activation, lysosomal $\mathrm{Ca}^{2+}$ release, and induction of TFEB-nuclear translocation [23].

TFEB promotes both autophagosome and lysosome biogenesis [24]. Lysosomal $\mathrm{Ca}^{2+}$ may also directly regulate lysosome biogenesis and autophagosome-lysosome fusion [23]. The present study showed that increased levels of mROS levels caused by UQCRB overexpression may be linked to lysosomal and autophagic pathways through TFEB. In mutant UQCRB-expressing cells, an increase in mROS levels led to TFEB-nuclear translocation and promoted lysosomal biogenesis (Fig. 4D). Likewise, in HCT116 cells, lysosomal activity decreased after treatment with a UQCRB inhibitor, and lysosomal biogenesis was reduced by mROS regulation (Fig. 6). Thus, the increase in mROS levels by the overexpression of UQCRB very likely could regulate the TFEB-lysosome biogenesis pathway [28,34].

ROS plays a significant role in autophagy by activating signaling pathways [35]. Tumor necrosis factor- $\alpha$ has been reported to induce autophagic cell death through a ROS-dependent mechanism [3]. Furthermore, many cancer cells have high levels of ROS and high frequencies of signaling 
events and mutations that increase ROS levels [3,36]. Mutations in mitochondrial DNA-encoded electron transport chain proteins have been reported in many types of human tumors [37]. For example, heteroplasmic mutations in complex I have been shown to increase mROS levels, colony formation rates in soft agar, and tumor formation in vivo [38]. These findings suggest that cancer cells show increased ROS production, which activates localized pro-tumorigenic signaling [3].

HCT 116 CRC cells, which naturally overexpress UQCRB, were used to validate this finding. The overexpression of this protein resulted in the production of higher levels of mROS than control cells, and subsequently affected autophagy (Fig. 1). Treating the cells with A1938, a UQCRB inhibitor, significantly reduced autophagy, and serum starvation led to cell death of HCT116 cells (Fig. 6). Under conditions that mimicked the physiology of humans, an increase in mROS levels may act as a survival signal to trigger autophagy to improve the quality of various physiological control mechanisms. However, the present study demonstrated that UQCRB activity reduced mROS production in HCT116 cells, and that nutrient starvation made HCT116 cells more susceptible to A1938-induced cell death [18].

Moreover, HCT116 cells were xenografted into mice that were randomly administered either a vehicle control or $10 \mathrm{mg} / \mathrm{kg}$ A1938. The tumor volumes of mice in the A1938 group decreased more than those of mice in the vehicle group, whereas their body weights remained unchanged (Supplementary Fig. 3). In this in vivo study, we found that A1938 administration attenuated tumor growth. Thus, A1938 might be a good candidate for inducing tumor regression.

Although the role of autophagy in cancer cells remains controversial, we demonstrated that autophagy contributed to tumor cell survival in CRC $[15,16]$. Therefore, autophagy inhibitors are often included in chemotherapeutic drug regimens for the treatment of cancers [39]. Autophagic flux of HCT116 cells significantly increased in response to nutrient stress, and autophagy inhibition was more sensitive in vivo than in normal colon cells [15]. These findings demonstrated that blocking autophagy decreased tumor growth (Supplementary Fig. 3). UQCRB variants play important roles in various cancers, such as hepatocellular carcinoma, ovarian cancer, and pancreatic ductal adenocarcinoma [40-43]. It would therefore be important to investigate how UQCRB is related to the pathophysiological roles of autophagy during tumorigenesis.

\section{Materials and Methods}

\subsection{Compounds and antibodies}

A1938 was synthesized by our group [11]. DMSOe (D2650), bafilomycin A1 (B1793), CQ (C6628), rapamycin (553210), mito-TEMPO, AO (A6014), and Triton X-100 were purchased from Sigma-Aldrich (St. Louis, MO). LysoTracker Deep Red (L12492), Hoechst33342 (H3570), Lipofectamine 2000 (52887), Plus Reagent (10964), protease, phosphatase inhibitor solution (78441), Dulbecco's Modified Eagle's Medium (DMEM), Roswell Park Memorial Institute Medium (RPMI), fetal bovine serum (FBS), and antibiotics were purchased from Invitrogen Thermo Fisher Scientific (Grand Island, NY). Anti-UQCRB was obtained from Sigma-Aldrich; anti-LC3B, anti-actin, and anti-LAMP1 were obtained from Abcam (Cambridge, UK). Anti-p62 was obtained from BD Biosciences (Franklin Lakes, NJ); and anti-TFEB and anti-GAPDH were from Cell Signaling Technology (Danvers, MA). Anti-Lamin A/C was obtained from Santa Cruz Biotechnology (Dallas, TX). The mRFP-GFP-LC3B plasmids were kindly provided by Dr. 
Jaewhan Song of Yonsei University (Seoul, Republic of Korea).

\subsection{Cell culture}

Control HEK293 (normal human kidney cells), MT, and CCD18co cells were grown in DMEM, supplemented with $10 \%$ FBS and 1\% antibiotics. HCT116 cells were grown in RPMI1640 with the same conditions as described above. All cells were incubated at $37^{\circ} \mathrm{C}$ in a humidified incubator with $5 \% \mathrm{CO}_{2}$ at a $\mathrm{pH}$ of 7.4. To maintain the stability of the mutant cell lines, $1 \mathrm{mg} / \mathrm{mL} \mathrm{G} 418$ was added to the medium on a regular basis.

\subsection{Immunoblotting}

Soluble proteins were harvested from cells using SDS lysis buffer comprised of $50 \mathrm{mM}$ Tris $\mathrm{HCl}$ with a $\mathrm{pH}$ of 6.8 , containing $10 \%$ glycerol, $2 \%$ SDS, $10 \mathrm{mM}$ dithiothreitol, and $0.005 \%$ bromophenol blue. Equal concentrations of proteins were separated by $11 \%$ or $12.5 \%$ SDS-PAGE and transferred to polyvinylidene fluoride membranes (EMD Millipore, Billerica, MA, USA). Blots were then blocked and immunolabeled overnight at $4{ }^{\circ} \mathrm{C}$ with the following primary antibodies: anti-UQCRB (Sigma-Aldrich), anti-LC3B, anti-actin, anti-LAMP1 (all from Abcam), anti-p62 (BD Biosciences), anti-TFEB and anti-GAPDH (Cell Signaling Technology), and antiLamin A/C (Santa Cruz Biotechnology). Immunolabeling was visualized using an enhanced chemiluminescence kit (Amersham Life Science, Chalfont, UK) according to the manufacturer's instructions. Images were quantified using Image Lab software (Bio-Rad, Hercules, CA). Actin and GAPDH were used as internal controls. All band intensities were proportional to the amount of target protein on the membrane as determined by the linear range of detection. Images were quantified using Image LabTM software (Bio-Rad).

\subsection{Immunocytochemistry}

To determine changes in autophagy, both WT HEK293- and mutant UQCRB-expressing cell lines were seeded at a density of $1.5 \times 10^{5}$ cells/well in 6-well plates and incubated overnight. The medium was then changed to serum-free DMEM (Gibco, Rockville, MD) and incubated for $24 \mathrm{~h}$. After starvation, the cell samples were fixed with 4\% formaldehyde (Sigma-Aldrich), diluted in phosphate-buffered saline (PBS) and washed with $1 \times$ PBS three times. The cells were then treated with LC3 primary antibody (Abcam) for $2 \mathrm{~h}$. Nuclei were stained with Hoechst (Invitrogen, Grand Island, NY). Images were obtained using an LSM980 confocal microscope at 400× magnification.

\section{5. mROS measurements}

Mitochondria ROS levels were assessed using MitoSOX red fluorescence mitochondrial superoxide indicator (Invitrogen). The cells were incubated with $5 \mu \mathrm{M}$ MitoSOX and Hoechst33342 (Life Technologies, Grand Island, NY) for 10 min, washed with medium or $1 \times$ PBS three times, and fixed with 4\% formaldehyde. MitoSOX and Hoechst staining results were analyzed using a Zeiss LSM 980 confocal microscope (Carl Zeiss MicroImaging, Thornwood, $\mathrm{NY}$ ), and the fluorescence intensity of MitoSOX was measured using ImageJ software (National Institutes of Health, Bethesda, MD). 


\subsection{The $m R F P-G F P-L C 3 B$ plasmid transfection}

Cells were transfected with the mRFP-GFP-LC3B plasmid using Lipofectamine 2000 transfection reagent (Invitrogen) for $24 \mathrm{~h}$. The cells were then treated with DMSO control, A1938, or mitoTEMPO for $24 \mathrm{~h}$. Nuclei were stained with Hoechst33342. Following incubation for $10 \mathrm{~min}$, the cells were fixed with $4 \%$ formaldehyde and washed three times with medium or PBS. Images were obtained using an LSM700 confocal microscope at 400× magnification.

\subsection{GCaMP3-ML1 Ca ${ }^{2+}$ imaging}

Cells were grown on $15 \mathrm{~mm}$ coverslips and transfected with a plasmid encoding a lysosomal GCaMP3-ML1 $\mathrm{Ca}^{2+}$ probe. The experiment was conducted $3-5 \mathrm{~h}$ after plating while cells still exhibited a round morphology. Lysosomal $\mathrm{Ca}^{2+}$ release was measured in a basal $\mathrm{Ca}^{2+}$ solution containing $145 \mathrm{mM} \mathrm{NaCl}, 5 \mathrm{mM} \mathrm{KCl}, 3 \mathrm{mM} \mathrm{MgCl} 2,10 \mathrm{mM}$ glucose, $1 \mathrm{mM}$ EGTA, and $20 \mathrm{mM}$ HEPES ( $\mathrm{pH}$ 7.4) with or without $40 \mu \mathrm{M}$ ML-SA1 and $1 \mu \mathrm{M}$ ionomycin by monitoring the fluorescence intensity at $470 \mathrm{~nm}$ with an LSM980 confocal microscope (Zeiss, Wetzlar, Germany).

\subsection{EGFP-TFEB nuclear translocation assay}

HEK293 and MT cells were seeded into 6-well plates and incubated overnight, and the enhanced green fluorescence protein-TFEB vector was then transfected into the cells using Lipofectamine 2000 reagent (Invitrogen) according to the manufacturer's instructions. Then, A1938 treatment was conducted for $6 \mathrm{~h}$ as indicated. Cells were then fixed with $4 \%$ formaldehyde and washed three times with medium or PBS. Nuclei were stained with Hoechst33342. Images were obtained using a LSM700 confocal microscope at $400 \times$ magnification.

\subsection{Fluorescence staining}

For AO staining, HEK293 and MT cells were grown on $15 \mathrm{~mm}$ coverslips at a density of $1.3 \times 10^{5}$ cells/well in 6-well plates. The cells were then treated with drugs for $24 \mathrm{~h}$, and then treated with 5 $\mu \mathrm{g} / \mathrm{mL}$ AO (Sigma-Aldrich). Nuclei were stained with Hoechst. The cells were then incubated for 20 min, fixed with 4\% paraformaldehyde (PFA), and washed three times with PBS. Images were obtained using an LSM880 confocal microscope at 400 × magnification. Red fluorescence intensity was quantified using ImageJ software. For LysoTracker Deep Red (L12492) staining, HEK293 and MT cells were grown on $15 \mathrm{~mm}$ coverslips at a density of $1.3 \times 10^{5}$ cells/well in 6 -well plates The cells were then treated with drugs for $24 \mathrm{~h}$, then treated with $100 \mathrm{nM}$ LysoTracker Deep Red for 20 min, fixed with 4\% PFA, and washed three times with medium or PBS. Images were obtained using an LSM700 confocal microscope at 400× magnification. The number of cell red puncta were quantified using ImageJ software.

\subsection{Cell proliferation assay}

Cell growth was measured using the MTT colorimetric assay. HCT116 cancer cells were seeded at a density of $5 \times 10^{3}$ cells/well in 96-well plates and incubated overnight. Cells were then treated with A1938 for various concentrations and durations. At the end of the assay, $2 \mathrm{mg} / \mathrm{mL}$ MTT was added to each well and incubated for $4 \mathrm{~h}$. MTT-formazan in each well was dissolved in $150 \mu \mathrm{L}$ 
DMSO, and the absorbance was read at $540 \mathrm{~nm}$ with a microplate reader (Bio-Tek Instruments, Winooski, VT)

\subsection{Cell death assay}

HCT116 cells were seeded at $2 \times 10^{4}$ cells/well in 24 -well plates and incubated overnight. The medium was then changed to serum-free DMEM (Gibco), and an inhibitor of cell death (CQ) was added. After $72 \mathrm{~h}$, cell viability was determined using trypan blue staining.

\subsection{Statistics}

All data are expressed as the mean $\pm \mathrm{SD}$ or mean $\pm \mathrm{SEM}$, as determined using Prism software, version 9.00 for Windows (GraphPad Software, San Diego, CA). Statistical analyses were performed using an unpaired, two-tailed Student's $t$-test. A value of $\mathrm{P}<0.05$ was considered statistically significant.

\section{Conclusion}

This study showed that UQCRB overexpression-induced autophagy may occur via generated mROS and that UQCRB is a potential therapeutic target for autophagy regulation in UQCRBrelated diseases, such as CRC.

This study determined the UQCRB protein expression and mROS generation levels of mutant UQCRB-expressing cell lines and HCT116 cells. UQCRB overexpression promoted autophagy flux by lysosomal $\mathrm{Ca}^{2+}$ release through the TRPML1 channel, which led to TFEB translocation into the nucleus to promote both autophagosome and lysosome biogenesis. Additionally, autophagy and lysosomal activity induced by UQCRB overexpression were abolished by the UQCRB inhibitor, A1938, in both MT and HCT116 cells. A1938-induced HCT116 cell death occurred in serum-free conditions that mimicked nutrient-deprived environments. In addition, the HCT116 xenograft study showed that A1938 treatment decreased tumor volume but did not affect the body weights of mice.

Collectively, the results demonstrated that UQCRB, a crucial mediator of mROS generation, was a potential therapeutic target for autophagy regulation. Small molecules that specifically regulate the function of UQCRB could suppress tumor growth by regulating the functional links between UQCRB, mROS, and autophagy. Therefore, UQCRB inhibitors could be a novel therapeutic strategy for regulating autophagy in the tumor environment.

\section{Supplementary Materials}

Figure S1: The UQCRB-overexpressing CRC cell line and wild-type HCT116 cells were sensitized during A1938-induced autophagy regulation.

Figure S2: A1938 suppressed autophagy and lysosomal activity in HCT116 cells.

Figure S3: The UQCRB inhibitor A1938 attenuated HCT116 xenograft tumor growth. 
Author Contributions: EJG, DSK, and HJK participated in the conception of the project and experimental design. EJG performed cell and molecular biology assays and analyzed the data. HYH performed the tumor xenograft mouse model assay and analyzed the data. EJG and HJK wrote the paper. All authors edited and approved the final manuscript.

Funding: This work was partly supported by grants from the National Research Foundation of Korea and was funded by the government of the Republic of Korea (MSIP; 2021R1A3B1077371, 2015K1A1A2028365, and 2012M3A9D1054520) and the Brain Korea 21 Plus Project, Republic of Korea and Institute of Convergence Science at Yonsei University.

Conflicts of Interest: The authors declare that they have no conflict of interest. 


\section{Graphical abstract}
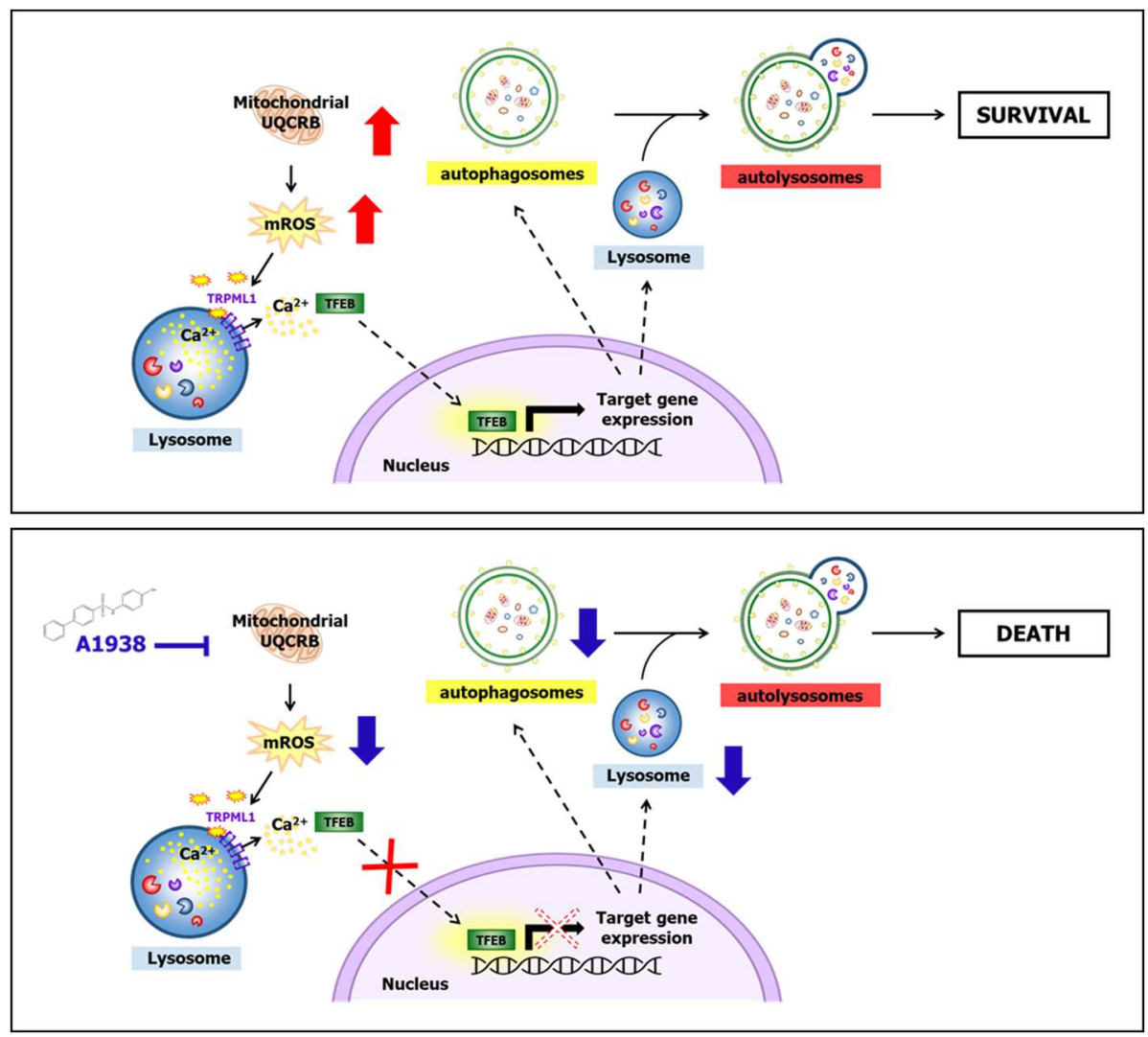


\section{References}

1. Porporato, P. E.; Filigheddu, N.; Bravo-San Pedro, J. M.; Kroemer, G.; Galluzzi, L., Mitochondrial metabolism and cancer. Cell research 2018, 28, 265-280.

2. Rigoulet, M.; Yoboue, E. D.; Devin, A., Mitochondrial ROS generation and its regulation: mechanisms involved in $\mathrm{H} 2 \mathrm{O} 2$ signaling. Antioxidants \& redox signaling 2011, 14, 459-468.

3. Sullivan, L. B.; Chandel, N. S., Mitochondrial reactive oxygen species and cancer. Cancer \& metabolism 2014, 2, 1-12.

4. Sabharwal, S. S.; Schumacker, P. T., Mitochondrial ROS in cancer: initiators, amplifiers or an Achilles' heel? Nature Reviews Cancer 2014, 14, 709-721.

5. Jung, H. J.; Shim, J. S.; Lee, J.; Song, Y. M.; Park, K. C.; Choi, S. H.; Kim, N. D.; Yoon, J. H.; Mungai, P. T.; Schumacker, P. T.; Kwon, H. J., Terpestacin inhibits tumor angiogenesis by targeting UQCRB of mitochondrial complex III and suppressing hypoxiainduced reactive oxygen species production and cellular oxygen sensing. Journal of Biological Chemistry 2010, 285, 11584-11595.

6. Suzuki, H.; Hosokawa, Y.; Toda, H.; Nishikimi, M.; Ozawa, T., Cloning and sequencing of a cDNA for human mitochondrial ubiquinone-binding protein of complex III. Biochemical and biophysical research communications 1988, 156, 987-994.

7. Sung, H.; Ferlay, J.; Siegel, R. L.; Laversanne, M.; Soerjomataram, I.; Jemal, A.; Bray, F., Global cancer statistics 2020: GLOBOCAN estimates of incidence and mortality worldwide for 36 cancers in 185 countries. CA: a cancer journal for clinicians 2021, 71, 209249.

8. Kim, H.-C.; Chang, J.; Lee, H. S.; Kwon, H. J., Mitochondrial UQCRB as a new molecular prognostic biomarker of human colorectal cancer. Experimental \& molecular medicine 2017, 49, e391-e391.

9. Hong, J. W.; Kim, J. M.; Kim, J. E.; Cho, H.; Kim, D.; Kim, W.; Oh, J.-W.; Kwon, H. J., MiR-4435 is an UQCRB-related circulating miRNA in human colorectal cancer. Scientific reports 2020, 10, 1-11.

10. Chang, J.; Jung, H. J.; Jeong, S. H.; Kim, H. K.; Han, J.; Kwon, H. J., A mutation in the mitochondrial protein UQCRB promotes angiogenesis through the generation of mitochondrial reactive oxygen species. Biochemical and biophysical research communications 2014, 455, 290-297.

11. Jung, H. J.; Cho, M.; Kim, Y.; Han, G.; Kwon, H. J., Development of a Novel Class of Mitochondrial Ubiquinol-Cytochrome c Reductase Binding Protein (UQCRB) Modulators as Promising Antiangiogenic Leads. Journal of medicinal chemistry 2014, 57, 7990-7998.

12. Chang, J.; Jung, H. J.; Park, H.-J.; Cho, S.-W.; Lee, S.-K.; Kwon, H. J., Cellpermeable mitochondrial ubiquinol-cytochrome $\mathrm{c}$ reductase binding protein induces angiogenesis in vitro and in vivo. Cancer letters 2015, 366, 52-60.

13. Jung, N.; Kwon, H. J.; Jung, H. J., Downregulation of mitochondrial UQCRB inhibits cancer stem cell-like properties in glioblastoma. International journal of oncology 2018, 52, 241-251.

14. Lin, J.; Chuang, C.-C.; Zuo, L., Potential roles of microRNAs and ROS in colorectal cancer: diagnostic biomarkers and therapeutic targets. Oncotarget 2017, 8, 17328.

15. Lauzier, A.; Normandeau-Guimond, J.; Vaillancourt-Lavigueur, V.; Boivin, V.; 
Charbonneau, M.; Rivard, N.; Scott, M. S.; Dubois, C. M.; Jean, S., Colorectal cancer cells respond differentially to autophagy inhibition in vivo. Scientific reports 2019, 9, 1-16.

16. Sato, K.; Tsuchihara, K.; Fujii, S.; Sugiyama, M.; Goya, T.; Atomi, Y.; Ueno, T.; Ochiai, A.; Esumi, H., Autophagy is activated in colorectal cancer cells and contributes to the tolerance to nutrient deprivation. Cancer research 2007, 67, 9677-9684.

17. Wu, Y. C.; Wu, W. K. K.; Li, Y.; Yu, L.; Li, Z. J.; Wong, C. C. M.; Li, H. T.; Sung, J. J. Y.; Cho, C. H., Inhibition of macroautophagy by bafilomycin A1 lowers proliferation and induces apoptosis in colon cancer cells. Biochemical and biophysical research communications 2009, 382, 451-456.

18. Fu, Y.; Hong, L.; Xu, J.; Zhong, G.; Gu, Q.; Gu, Q.; Guan, Y.; Zheng, X.; Dai, Q.; Luo, X., Discovery of a small molecule targeting autophagy via ATG4B inhibition and cell death of colorectal cancer cells in vitro and in vivo. Autophagy 2019, 15, 295-311.

19. Chen, C.; Deng, M.; Sun, Q.; Loughran, P.; Billiar, T. R.; Scott, M. J., Lipopolysaccharide stimulates p62-dependent autophagy-like aggregate clearance in hepatocytes. BioMed research international 2014, 2014.

20. Mizushima, N.; Murphy, L. O., Autophagy assays for biological discovery and therapeutic development. Trends in Biochemical Sciences 2020.

21. Mauthe, M.; Orhon, I.; Rocchi, C.; Zhou, X.; Luhr, M.; Hijlkema, K.-J.; Coppes, R. P.; Engedal, N.; Mari, M.; Reggiori, F., Chloroquine inhibits autophagic flux by decreasing autophagosome-lysosome fusion. Autophagy 2018, 14, 1435-1455.

22. Zhou, C.; Zhong, W.; Zhou, J.; Sheng, F.; Fang, Z.; Wei, Y.; Chen, Y.; Deng, X.; Xia, B.; Lin, J., Monitoring autophagic flux by an improved tandem fluorescent-tagged LC3 (mTagRFP-mWasabi-LC3) reveals that high-dose rapamycin impairs autophagic flux in cancer cells. Autophagy 2012, 8, 1215-1226.

23. Zhang, X.; Cheng, X.; Yu, L.; Yang, J.; Calvo, R.; Patnaik, S.; Hu, X.; Gao, Q.; Yang, M.; Lawas, M., MCOLN1 is a ROS sensor in lysosomes that regulates autophagy. Nature communications 2016, 7, 1-12.

24. Medina, D. L.; Di Paola, S.; Peluso, I.; Armani, A.; De Stefani, D.; Venditti, R.; Montefusco, S.; Scotto-Rosato, A.; Prezioso, C.; Forrester, A., Lysosomal calcium signalling regulates autophagy through calcineurin and TFEB. Nature cell biology 2015, 17, 288-299.

25. Martina, J. A.; Puertollano, R., Rag GTPases mediate amino acid-dependent recruitment of TFEB and MITF to lysosomes. Journal of Cell Biology 2013, 200, 475-491.

26. Scherz-Shouval, R.; Shvets, E.; Fass, E.; Shorer, H.; Gil, L.; Elazar, Z., Reactive oxygen species are essential for autophagy and specifically regulate the activity of Atg4. The EMBO journal 2007, 26, 1749-1760.

27. Pierzyńska-Mach, A.; Janowski, P. A.; Dobrucki, J. W., Evaluation of acridine orange, LysoTracker Red, and quinacrine as fluorescent probes for long-term tracking of acidic vesicles. Cytometry Part A 2014, 85, 729-737.

28. Settembre, C.; Fraldi, A.; Medina, D. L.; Ballabio, A., Signals from the lysosome: a control centre for cellular clearance and energy metabolism. Nature reviews Molecular cell biology 2013, 14, 283-296.

29. Cheng, X.-T.; Xie, Y.-X.; Zhou, B.; Huang, N.; Farfel-Becker, T.; Sheng, Z.-H., Revisiting LAMP1 as a marker for degradative autophagy-lysosomal organelles in the 
nervous system. Autophagy 2018, 14, 1472-1474.

30. Klimova, T.; Chandel, N., Mitochondrial complex III regulates hypoxic activation of HIF. Cell death and differentiation 2008, 15, 660.

31. Jung, H. J.; Kim, Y.; Chang, J.; Kang, S. W.; Kim, J. H.; Kwon, H. J., Mitochondrial UQCRB regulates VEGFR2 signaling in endothelial cells. Journal of Molecular Medicine 2013, 91, 1117-1128.

32. Jian, F.; Chen, D.; Chen, L.; Yan, C.; Lu, B.; Zhu, Y.; Chen, S.; Shi, A.; Chan, D. C.; Song, Z., Sam50 regulates PINK1-Parkin-mediated mitophagy by controlling PINK1 stability and mitochondrial morphology. Cell reports 2018, 23, 2989-3005.

33. Lu, X.; Xuan, W.; Li, J.; Yao, H.; Huang, C.; Li, J., AMPK protects against alcoholinduced liver injury through UQCRC2 to up-regulate mitophagy. Autophagy 2021, 1-22.

34. Li, L.; Tan, J.; Miao, Y.; Lei, P.; Zhang, Q., ROS and autophagy: interactions and molecular regulatory mechanisms. Cellular and molecular neurobiology 2015, 35, 615-621.

35. Scherz-Shouval, R.; Elazar, Z., ROS, mitochondria and the regulation of autophagy. Trends in cell biology 2007, 17, 422-427.

36. Weinberg, F.; Hamanaka, R.; Wheaton, W. W.; Weinberg, S.; Joseph, J.; Lopez, M.; Kalyanaraman, B.; Mutlu, G. M.; Budinger, G. S.; Chandel, N. S., Mitochondrial metabolism and ROS generation are essential for Kras-mediated tumorigenicity. Proceedings of the National Academy of Sciences 2010, 107, 8788-8793.

37. Chatterjee, A.; Mambo, E.; Sidransky, D., Mitochondrial DNA mutations in human cancer. Oncogene 2006, 25, 4663-4674.

38. Park, J. S.; Sharma, L. K.; Li, H.; Xiang, R.; Holstein, D.; Wu, J.; Lechleiter, J.; Naylor, S. L.; Deng, J. J.; Lu, J., A heteroplasmic, not homoplasmic, mitochondrial DNA mutation promotes tumorigenesis via alteration in reactive oxygen species generation and apoptosis. Human molecular genetics 2009, 18, 1578-1589.

39. Karantza-Wadsworth, V.; Patel, S.; Kravchuk, O.; Chen, G.; Mathew, R.; Jin, S.; White, E., Autophagy mitigates metabolic stress and genome damage in mammary tumorigenesis. Genes \& development 2007, 21, 1621-1635.

40. Kim, J. E.; Hong, J. W.; Lee, H. S.; Kim, W.; Lim, J.; Cho, Y. S.; Kwon, H. J., HsamiR-10a-5p downregulation in mutant UQCRB-expressing cells promotes the cholesterol biosynthesis pathway. Scientific reports 2018, 8, 1-10.

41. Jia, H.-L.; Ye, Q.-H.; Qin, L.-X.; Budhu, A.; Forgues, M.; Chen, Y.; Liu, Y.-K.; Sun, H.-C.; Wang, L.; Lu, H.-Z., Gene expression profiling reveals potential biomarkers of human hepatocellular carcinoma. Clinical Cancer Research 2007, 13, 1133-1139.

42. Wrzeszczynski, K. O.; Varadan, V.; Byrnes, J.; Lum, E.; Kamalakaran, S.; Levine, D. A.; Dimitrova, N.; Zhang, M. Q.; Lucito, R., Identification of tumor suppressors and oncogenes from genomic and epigenetic features in ovarian cancer. PloS one 2011, 6, e28503.

43. Harada, T.; Chelala, C.; Crnogorac-Jurcevic, T.; Lemoine, N. R., Genome-wide analysis of pancreatic cancer using microarray-based techniques. Pancreatology 2009, 9, 13-24. 